\title{
Pilot's visual attention allocation modeling under fatigue
}

\author{
$\mathrm{Xu} \mathrm{Wu}$, Xiaoru Wanyan and Damin Zhuang* \\ Department of Aeronautics Science and Engineering, Beijing University of Aeronautics and \\ Astronautics, Beijing, China
}

\begin{abstract}
.
BACKGROUND: Human factors involved with visual attention mechanism and fatigue are critical causes of modern aviation accidents.

OBJECTIVE: To investigate the connection between attention and flight fatigue, a mathematical model of pilot's visual attention allocation was established based on information processing channels. Multi-task condition and current psychophysical state were taken into account as well.

METHODS: Sixteen participants were recruited to perform a long-term dual-task in a Boeing 737-800 flight simulator. The primary task was an envelope flight task and the secondary was an unusual attitude (UA) recovery task. Reaction time of the secondary task was recorded as a behavior performance index, while heart rate and respiration rate were measured as physiological indices as well as fixation distribution as attention allocation index.

RESULTS: The experiment results showed a significant affect of experiment time that indicated the occurrence and influence of fatigue. Eye movement tracking also revealed good agreement with the predictable model and hence verified its effectiveness. Moreover, applicability of the model was validated under flight fatigue and multiple tasks condition.

CONCLUSION: The current study provided a quantitative connection between pilot's visual attention allocation and flight fatigue, which was verified in the ergonomics experiment.
\end{abstract}

Keywords: Visual attention allocation, mathematical model, fatigue, ergonomics experiment

\section{Introduction}

As a traditional and classic topic in cognitive psychology and neuroscience, attentional capture is capable of being a limited resource allocation and endows perception with the ability to select. With the development of automatics and informatics, visual attention plays a significant role even in aviation. At this time, attention allocation, mental workload and situation awareness are considered as the popular human factors in aviation, which have direct impact on flight performance and safety [1].

Currently, cognitive modeling becomes a major method to investigate the essence of pilots' attention allocation [2]. Senders established one of the first quantitative models of indicator-monitoring behavior by introducing the concept of bandwidth, which suggested the relationship between visual scan and the bandwidth of indicator information [3]. Subsequently, Carbonell and Sheridan considered the influence of information value and improved Senders' model [4]5]. Wickens attributed attention allocation to four

\footnotetext{
${ }^{*}$ Corresponding author: Damin Zhuang, Department of Aeronautics Science and Engineering, Beijing University of Aeronautics and Astronautics, Beijing, China. Tel.: +86 010 82338163; E-mail: dmzhuang@ buaa.edu.cn.
} 
impact factors and founded a salience-effort-expectancy-value (SEEV) model to predict the visual scan behavior. In addition, SEEV was promoted into Noticing-SEEV (N-SEEV), a model used to analyze the ability of attention capture for an emergency [6]. Moreover, Wanyan et al. applied fuzzy mathematics to quantify the cognitive process of indicator information priority. The researchers proposed a hybrid entropy model to predict attention allocation which involved three main factors: information priority, potential probability and detection efficiency [7]. However, there were still limitations when considering the quantitative basis and other influence factors. Therefore, multi-factor model was established and calculated on the basis of SEEV and hybrid entropy models [8]. The multi-factor model rearranged the factors in SEEV and provided a detailed quantitation. However, it only focused on single task situation and failed to discuss the influence of workload in validation.

Unfortunately, attention is hard to maintain since it can be degraded by vigilance and fatigue [9]. Fatigue-related human error in the cockpit may lead to crew-member and passenger fatalities as well as the loss of the airframe itself. However, physiological tracking method could examine the appearance of fatigue and make alert, it was still used in the laboratory research. Furthermore, for military pilots, fatigue may cause a failure to acquire, engage and destroy enemy targets, or worse, can result in incorrect targeting and destruction of non-threatening assets. Flight fatigue is produced predominantly by crew scheduling and workload factors that curtail sleep and increase stress [10]. Nevertheless, the flight fatigue effect on visual attention allocation is rarely studied and lacks more theoretical and experimental evidence. Besides, comprehensive measurement of eye-movement and physiological indices provided a useful and challenging method to study the relationship between attention and fatigue.

In order to explore the connection between fatigue and attention, a mathematical model concerned with multiple task situations and current psychophysical state was proposed to predict visual attention allocation based on a multi-factor model. To verify this model under fatigue conditions, an ergonomics experiment was carried out in a flight simulator with dual tasks. Flight simulation tasks were performed to induce flight fatigue and to measure fixation, pupil diameter, heart rate, as well as other physiological indices. The experiment results were processed in descriptive statistics, analysis of variance and regression analysis.

\section{Mathematical modeling of attention allocation}

Assuming the percentage attention resource allocated to $n$ areas of interest (AOIs) was:

$$
p=\left(p_{1}, \ldots, p_{i}, \ldots, p_{n}\right)^{T}, T \text { for vector transpose. }
$$

Generally, the procedure of information processing was involved with two channels: top-down and bottom-up. The top-down channel was part of an automatic search or selective control in the visual system while the bottom-up channel was driven by visual features of obtained information. Based on the multi-factor model, both channels could be quantified as the interaction of four influencing factors [8]. For most human-machine interaction systems in aviation, those factors included occurring probability $P$, information priority $V$, effort $E$, and salience $S$. These factors simultaneously affected and determined a pilot's attention allocation mechanism. Therefore, an m-dimension linear space $A$ was spanned by these $m$ factors. The information state obtained in AOI could be expressed as the matrix $A_{n \times m}$ in space $A$, where $a_{i j} \in A_{n \times m}$ stood for the coefficient of $\mathrm{AOI}_{i}$ for dimension $j$. In order to simplify the model, the defined sum of coefficients of AOIs for single dimension was 1 :

$$
\sum_{i=1}^{n} a_{i j}=1, \text { for } j=1, \ldots, m .
$$


The cognitive resource of attention had the ability to identify and classify the stimulus [11]. The requirement of cognitive resource increased as the stimulus became complicated. Because of the limited cognitive resource, no more stimuli would be processed when the whole resource was occupied. Moreover, cognitive resource was closely related to attention mechanism and psychophysical state [12]. Therefore, the attention resource allocated to AOIs in Eq. (1) was advanced as:

$$
p=A \times b, \quad b=\left(b_{1}, \ldots, b_{j}, \ldots, b_{m}\right)^{T} .
$$

Where $b_{j}$ quantified the weighting of each dimension in $A$ according to the current psychophysical state and brain arousal level:

$$
\sum_{j=1}^{m} b_{j}=1
$$

In consideration of multiple flight tasks in aviation, the difficulties and priorities of tasks were still manipulated to drive pilot attention allocation. The weight of tasks was defined as $t_{k}$, therefore, $A$ was expanded into $A_{k}$ under different task conditions. The attention allocation was calculated as:

$$
p=\sum t_{k} p_{k}, \quad p_{i}=\sum t_{k} p_{k i}=\sum t_{k} \cdot \sum_{j=1}^{m} a_{k i j} b_{k j}, \text { for } i=1, \ldots, n .
$$

Specifically, as other possible factors had not been explicitly quantified by their impacts on attention mechanism, the multiple factors mentioned above were adopted to form a four-dimension subspace $A^{*}$ with the bases as $\{P, V, E, S\}$. In $A^{*}, p_{i}=\sum t_{k} \cdot \sum_{j=1}^{4} a_{k i j} b_{j}$ where $a_{k i j}$ represented the four dimensions of $\{P, V, E, S\}$ and $b_{j}$ represented their weights according to the current brain arousal level.

In addition, $a_{k i 1}$ indicated the occurring frequency and amount of obtained information which was reflected the concept of bandwidth in Senders' model [3]. Also, $a_{k i 2}$ could be determined as:

$$
a_{k i 2}=v_{k i} \cdot u_{k i} \text {. }
$$

Where $v_{k i}$ described the possibility of which potential cognitive state became available and $u_{k i}$ represented the cognitive evaluation of information priority. Since $u_{k i}$ was obscure and difficult to determine which was influenced by the physical and psychological state of the pilot, it was possible that even the most valuable information could be ignored and failed to be captured. Therefore, based on the hybrid entropy model [9], $v_{k i}$ could be optimized and calculated as:

$$
v_{k i}^{*}=\exp h_{k i} / \sum_{n} \exp h_{k i} .
$$

Where $h_{k i}$ was the binary fuzzy entropy of $\chi_{k i}$ :

$$
h_{k i}=-\chi_{k i} \ln \chi_{k i}-\left(1-\chi_{k i}\right) \ln \left(1-\chi_{k i}\right) .
$$

Where $\chi_{k i}$ was the fuzzy membership degree standardized into $[0,1]$ based on cognitive evaluation of $u_{k i}$. Therefore, the information priority $a_{k i 2}$ could be quantified as:

$$
a_{k i 2}^{*}=v_{k i}^{*} \cdot u_{k i} .
$$

Also, $a_{k i 3}$ illustrated the effort was consumed if eye or head movement was required to obtain visual information. It was measured by the distance between AOIs which showed visual attention was prone to transfer and allocated in the adjacent AOIs. Moreover, $a_{k i 4}$ was considered as the interaction of color, 


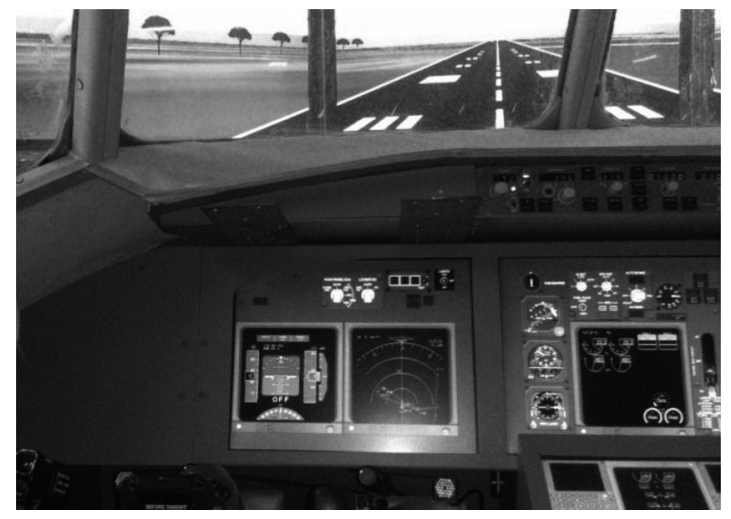

Fig. 1. The Boeing 737-800 flight simulator.

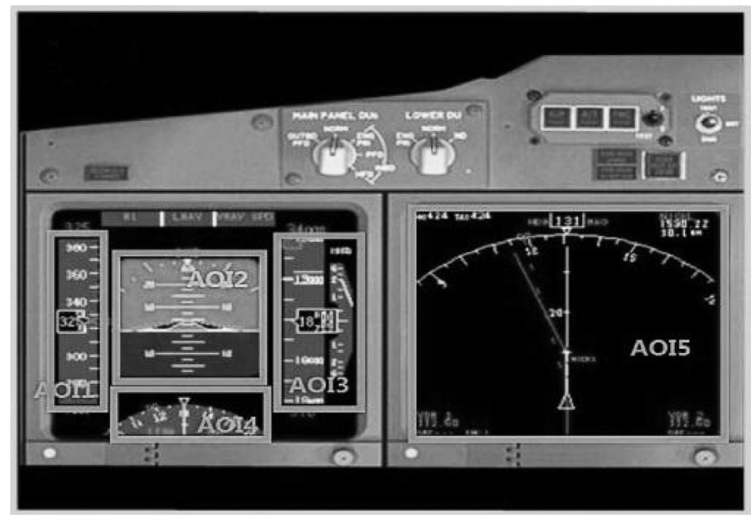

Fig. 2. Experiment interface.

size, along with character type, in the viewpoint of multiple visual coding of flight information in the cockpit's display interface [8].

\section{Methods}

\subsection{Apparatus}

The experiment was carried out in a Boeing 737-800 flight simulator as shown in Fig. 1. The experiment interface was designed as the simulation interface of the primary flight display (PFD) and navigation display (ND). Based on corneal reflection, Smart Eye Pro 5.9 was used to track the participant's eye movement in a natural way $(60 \mathrm{~Hz})$, and the fixation distribution, pupil diameter (PD) and eyelid opening (EO) were recorded [7]. A Tongfang Shenhuo TH-P physiological tester $(40 \mathrm{kHz})$ was utilized to measure the participant's skin electrodermal activity (EDA), heart rate (HR) and respiration rate (RespR) through sensors attached to their wrists and fingers of left hands [13].

\subsection{Participants}

Sixteen participants from Beihang University were recruited in this study. All participants (10 males, 6 females, and 23.4 years \pm 0.7 years old) were familiar with the basic aeronautics knowledge and flight simulator operations. All participants were right-handed with normal or correct to normal vision.

\subsection{Experiment task}

A dual flight task was performed in the experiment. The primary task was to manually fly according to the flight path shown on ND. The secondary task was an unusual attitude (UA) recovery task which required participants to monitor the status of target information. Once a target indicator exceeded the normal range, the unusual attitude appeared and the corresponding response of the steering wheel was expected to be taken to recover and eliminate UA. Five flight indicators of PFD were selected as the monitoring task target, including indicated airspeed, barometric altitude, pitching angle, rolling angle and heading angle. The experiment interface was divided into five AOIs as shown in Fig. 2. It should be noticed that the $\mathrm{AOI}_{2}$ contained two inseparable indicators of pitching and rolling angles. 


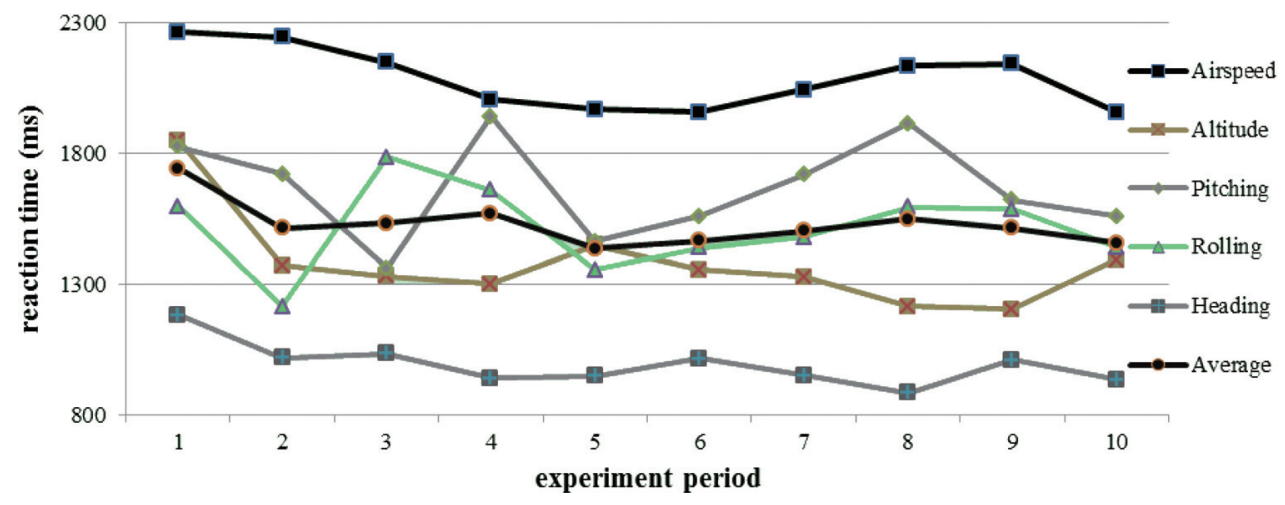

Fig. 3. The descriptive statistics of participant's reaction time of UA recovery task (ms).

\subsection{Experiment procedure}

Participants were asked to practice the dual-task operations until they were well trained. During the experiment, the participants were required to allocate their attention reasonably according to the different task conditions. The primary task was performed continuously while the secondary task intervened randomly. The whole experiment lasted for about 100 minutes, which was divided into ten periods for the analysis of flight fatigue. The secondary task occurred 50 times in total, with each indicator turning unusual once per period. Since UA was not recovered until the corresponding operation was performed, only reaction time was recorded automatically as behavior performance of the secondary task. Since the physiological sensors were attached to left fingers, participants were required to perform the dual-task using only their right hands. Smart Eye Pro 5.9 and the TH-P physiological tester kept real-time tracking. To specify, heart rate was measured through blood volume pulse (BVP).

\section{Results}

\subsection{The experimental results of behavior performances and physiological indices}

The descriptive statistics is adopted to show the average reaction time and physiological indices while the repeated measured ANOVA is adopted to analyze the main effect of flight indicator and experiment period on those indices. The descriptive statistics results of reaction time of UA recovery task during the entire experiment are shown in Fig. 3, and the participant's physiological indices are shown in Table 1.

A two-way repeated measured ANOVA of reaction time revealed insignificant main effect of experiment period $\left(F(9,135)=1.103, p=0.365, \eta^{2}=0.068\right)$ and significant main effect of flight indicator $\left(F(4,60)=20.483, p<0.001, \eta^{2}=0.577\right)$. The interaction between them was also insignificant $(F(36$, $\left.540)=0.654, p=0.942, \eta^{2}=0.042\right)$. Obviously, reaction time of indicated airspeed was much longer than that of the heading angle and the differences between those indicators varied a little during the experiment.

A one-way repeated measured ANOVA of five physiological indices revealed significant main effect of experiment period $\left(F_{P D}(9,135)=2.619, p_{P D}=0.008, \eta^{2}=0.149 ; F_{E O}(9,135)=2.851, p_{E O}=\right.$ $0.004, \eta^{2}=0.160 ; F_{H R}(9,135)=3.246, p_{H R}=0.001, \eta^{2}=0.178 ; F_{\text {RespR }}(9,135)=4.325, p_{\text {RespR }}<$ $\left.0.001, \eta^{2}=0.224 ; F_{E D A}(9,135)=1.496, p_{E D A}=0.155, \eta^{2}=0.091\right)$. In addition, both reaction time and physiological indices were influenced by experiment period. As mental and physical workload 
Table 1

The participant's physiological indices during different experiment periods $(\mathrm{M} \pm \mathrm{SD})$

\begin{tabular}{cccccc}
\hline Period & PD $(\mathrm{mm})$ & EO $(\mathrm{mm})$ & HR $(\mathrm{bpm})$ & RespR $(\mathrm{bpm})$ & EDA $(\mathrm{mv})$ \\
\hline 1 & $3.71 \pm 0.85$ & $10.26 \pm 1.91$ & $79.19 \pm 10.03$ & $19.03 \pm 3.02$ & $2.49 \pm 0.28$ \\
2 & $3.53 \pm 0.87$ & $10.11 \pm 1.75$ & $77.87 \pm 11.04$ & $18.40 \pm 2.93$ & $2.56 \pm 0.09$ \\
3 & $3.37 \pm 0.89$ & $10.03 \pm 1.87$ & $78.05 \pm 9.41$ & $18.24 \pm 2.76$ & $2.54 \pm 0.11$ \\
4 & $3.41 \pm 0.93$ & $9.78 \pm 1.81$ & $77.56 \pm 12.77$ & $17.76 \pm 2.55$ & $2.54 \pm 0.14$ \\
5 & $3.36 \pm 0.89$ & $9.71 \pm 1.76$ & $77.17 \pm 12.24$ & $17.38 \pm 2.45$ & $2.60 \pm 0.18$ \\
6 & $3.40 \pm 0.88$ & $9.63 \pm 1.65$ & $77.26 \pm 11.56$ & $17.25 \pm 2.13$ & $2.52 \pm 0.11$ \\
7 & $3.34 \pm 0.83$ & $9.80 \pm 1.44$ & $75.15 \pm 10.39$ & $17.16 \pm 2.18$ & $2.49 \pm 0.09$ \\
8 & $3.30 \pm 0.82$ & $9.86 \pm 1.28$ & $74.18 \pm 11.60$ & $17.44 \pm 1.91$ & $2.50 \pm 0.09$ \\
9 & $3.23 \pm 0.76$ & $9.78 \pm 1.33$ & $74.84 \pm 10.05$ & $17.19 \pm 1.92$ & $2.46 \pm 0.17$ \\
10 & $3.30 \pm 0.75$ & $9.66 \pm 1.54$ & $72.91 \pm 12.15$ & $17.18 \pm 1.96$ & $2.45 \pm 0.12$ \\
\hline
\end{tabular}

Table 2

Percentage fixation distribution of AOIs during different experiment periods ( $\mathrm{M} \pm \mathrm{SD}, \%)$

\begin{tabular}{ccccrr}
\hline Period & \multicolumn{1}{c}{ AOI 1 } & \multicolumn{1}{c}{ AOI 2 } & \multicolumn{1}{c}{ AOI 3 } & \multicolumn{1}{c}{ AOI 4 } & \multicolumn{1}{c}{ AOI 5 } \\
\hline 1 & $27.6 \pm 3.1$ & $35.5 \pm 4.2$ & $19.4 \pm 2.2$ & $11.1 \pm 1.7$ & $6.4 \pm 0.8$ \\
2 & $26.6 \pm 4.3$ & $38.1 \pm 4.8$ & $20.0 \pm 2.4$ & $8.3 \pm 1.2$ & $7.0 \pm 0.8$ \\
3 & $26.2 \pm 4.1$ & $35.4 \pm 4.3$ & $21.1 \pm 2.6$ & $8.2 \pm 1.1$ & $9.1 \pm 0.9$ \\
4 & $24.0 \pm 3.7$ & $35.1 \pm 4.3$ & $22.3 \pm 2.5$ & $7.7 \pm 1.1$ & $10.9 \pm 0.8$ \\
5 & $20.1 \pm 3.1$ & $22.0 \pm 3.1$ & $22.3 \pm 2.7$ & $18.9 \pm 1.7$ & $16.7 \pm 1.2$ \\
6 & $21.2 \pm 3.2$ & $22.9 \pm 3.4$ & $22.1 \pm 2.0$ & $16.9 \pm 1.5$ & $16.9 \pm 1.3$ \\
7 & $19.7 \pm 2.8$ & $22.9 \pm 3.0$ & $22.9 \pm 3.1$ & $18.2 \pm 1.8$ & $16.3 \pm 2.1$ \\
8 & $17.8 \pm 3.5$ & $25.3 \pm 4.1$ & $21.9 \pm 2.7$ & $18.9 \pm 1.5$ & $16.1 \pm 1.6$ \\
9 & $19.9 \pm 3.0$ & $24.2 \pm 3.7$ & $20.8 \pm 1.9$ & $17.1 \pm 1.6$ & $18.0 \pm 2.1$ \\
10 & $18.9 \pm 3.1$ & $23.4 \pm 2.9$ & $20.8 \pm 2.2$ & $19.1 \pm 2.1$ & $17.8 \pm 1.8$ \\
\hline
\end{tabular}

accumulated, the reduction of behavior performance and changes of physiological indices indicated the occurrence of fatigue.

\subsection{The predictable and experimental results of attention allocation}

Based on the model and experiment design mentioned above, the predicted results of attention allocation were calculated in space $A^{*}$ with $n=5, m=4$. Since dual tasks were adopted in the study, the task weight was set as $t_{1}=0.6, t_{2}=0.4$. Therefore, $A_{k}$ could be decided according to Eqs (2), (6)-(9):

$$
A_{1}=\left(\begin{array}{llll}
0.205 & 0.222 & 0.187 & 0.200 \\
0.251 & 0.333 & 0.225 & 0.247 \\
0.205 & 0.222 & 0.201 & 0.237 \\
0.133 & 0.111 & 0.131 & 0.226 \\
0.205 & 0.111 & 0.256 & 0.089
\end{array}\right), A_{2}=\left(\begin{array}{cccc}
0.250 & 0.200 & 0.187 & 0.200 \\
0.250 & 0.400 & 0.225 & 0.247 \\
0.250 & 0.200 & 0.201 & 0.237 \\
0.250 & 0.200 & 0.131 & 0.226 \\
0 & 0 & 0.256 & 0.089
\end{array}\right) .
$$

In the quantitatively weighting of each dimension in $A^{*}, b_{j}$ was affected by the current psychophysical state. Thus, $b_{\text {high }}=(0.4,0.4,0.1,0.1)^{T}, b_{\text {low }}=(0.1,0.1,0.4,0.4)^{T}$ as brain arousal level being high or low [10]. To predict attention allocation under fatigue, both $b_{\text {high }}$ and $b_{\text {low }}$ were used in the calculation. Hence, following $p_{i}=\sum t_{k} \cdot \sum_{j=1}^{4} a_{k i j} b_{k j}$, the predicted result of attention allocation was $p_{h i g h}=$ $(0.213,0.291,0.218,0.110)^{T}, p_{\text {low }}=(0.198,0.250,0.219,0.178,0.157)^{T}$.

The experimental results of attention allocation were measured as a percentage fixation distribution of AOIs, which was also calculated in different experiment periods and shown in Table 2. Moreover, the attention resource allocated on AOIs was quite different from experiment periods especially in $\mathrm{AOI}_{5}$. 


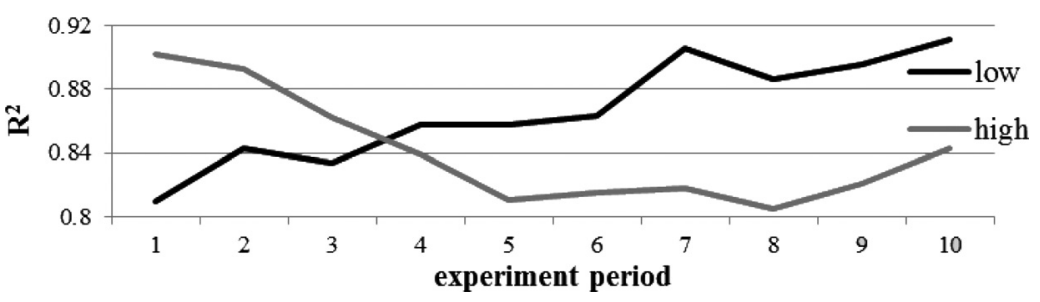

Fig. 4. Parameter $R^{2}$ of regression analysis.

The $\mathrm{AOI}_{5}$ of ND drew little attention resource at first but became increasingly attractive since fatigue appeared.

Regression analysis was used to examine the correlation between predicted and experimental results of attention allocation. The residuals $R^{2}$ of regression analysis in different periods was shown in Fig. 4 . Therefore, brain arousal level seemed to decline over time and the prediction of model at low level was better suited when fatigue occurred.

\section{Discussions}

In consideration of current psychophysical state and task priority, the predictable model of attention allocation was established and calculated based on a multi-factor model. In the validation under fatigue, an ergonomics experiment was carried out in a flight simulator with dual tasks, eye-movement, as well as physiological indices, being measured. The experimental results indicated the occurrence of fatigue and also revealed a correlation between predicted attention allocation and fixation distribution.

The analysis of behavior performances and physiological indices showed major sensitivity to the experimental period. Pupil diameter and eyelid opening respectively decreased over time, which reflected the accumulation of mental and physical workload [2]. However, pupil diameter and eyelid opening could indicate the current level of mental and physical workload. Once fatigue was induced by the accumulated workload, pupil diameter and eyelid opening would change and become smaller as deeper fatigue [14[15]. Also, heart rate, respiration rate and electrodermal activity also reduced as the psychophysical fatigue slowly appeared [16]. According to the tendency of the average reaction time in Fig. 3, the first occurrence of fatigue was supposed to be found at the half hour mark (period 3) and the second occurrence taking place around the one hour mark (period 6-7) because the average reaction time started to extend and became longer during those periods. Moreover, it proceeded to get faster after the occurrence of fatigue, which suggested an adaptation and recovery of fatigue.

The experimental result of the fixation distribution varied during all of periods. The $\mathrm{AOI}_{2}$ associated with the flight attitude indicator obtained most attention resource (22\% to $35 \%$ ), and $\mathrm{AOI}_{5}$ of the navigation display acquired the least (6\% to $18 \%)$. However, the gap between them seemed to decline as time went by. At the beginning of the experiment, $\mathrm{AOI}_{1}$ and $\mathrm{AOI}_{2}$ together attracted more than $60 \%$ of attention resource. Since participants were excited and concentrated on performing flight tasks, AOIs of high priority and task-relevance were prone to draw more attention. With the increase of both mental and physical workload, fatigue started to appear as they were accumulated until it reached a critical threshold. When participants felt increasingly tired, their brain arousal level dropped and remained low. Specifically during experiment period 4 and 5, the attention allocation of $\mathrm{AOI}_{2}$ sharply decreased from $35 \%$ to $22 \%$ while that of $\mathrm{AOI}_{5}$ increased from $10 \%$ to $16 \%$. The results explained how fatigue influenced and interacted with the attention allocation mechanism, which was consistent with the research literature [10]. 
Regression analysis was used to verify the effectiveness of predicted attention allocation, and was calculated under two brain arousal levels. Figure 4 showed that $R^{2}$ of $p_{\text {high }}$ was about 0.9 at first and then slowly decreased over time. On the contrary, $R^{2}$ of $p_{\text {low }}$ was unsatisfactory in the beginning but gradually increased above 0.9 during the final period. In addition, when fatigue first occurred, $R^{2}$ of two situations indicated a tendency of reversion, which was found around the intersection point of two lines. Attention tended to be allocated in the AOIs of high salience when brain arousal level decreased, which indicated the dominance of bottom-up factors. It was consistent with the viewpoint in Lavie's perceptual load theory that the level of processing which occurs for any one stimulus is dependent on the current perceptual load [17/18]. In addition, if the current task was attention-demanding and its processing exhausted all the available resources, little remained available to process other non-target stimulus in the visual field. Alternatively, if processing required a small amount of cognitive resources, perceptual load was low and attention was inescapably directed to the non-target stimulus [19]. That is, cognitive resource overflow could be used to process the irrelevant information at the low level of brain arousal, and thus, the salient AOI was more likely to draw attention.

Nevertheless, the current research concentrated on the establishment of attention allocation models and the computation method and validation experiment were carried out in order to provide a feasible way to complete the model. Furthermore, this study analyzed and discussed the eye movement tracking result of attention allocation. Other physiological indices and individual differences and their relationships to attention allocation and psychophysical fatigue would be analyzed in subsequent research.

\section{Acknowledgements}

This study was financially supported by National Basic Research Program of China (Grant No. 2010CB734104) and National Natural Science Foundation of China (Grant No. 61179053).

\section{References}

[1] Wiegmann DA, Shappell SA. Human error perspectives in aviation. Int J Aviat Psychol. 2001; 11(4): 341-357; doi: 10.1207/S15327108IJAP1104_2.

[2] Zhuang DM, Wanyan XR. Pilot attention allocation theory and applications. Science Press: Beijing; 2013.

[3] Senders JW. The human operator as a monitor and controller of multidegree of freedom systems. IEEE Trans Hum Electron. 1964; 5(1):2-5.

[4] Carbonell JR. A queueing model of many-instrument visual sampling. IEEE Trans Hum Electron. 1966; 7(4): 157-164.

[5] Sheridan TB. On how often the supervisor should sample. IEEE Trans Syst Scid Cybern. 1970; 6(2): 140-145; doi: 10.1109/TSSC.1970.300289.

[6] Wickens CD, McCarley JS, Steelman-Allen KS. NT-SEEV: A model of attention capture and noticing on the flight deck. Proc Hum Ergon Soc Annu Meet. 2009; 53(12): 769-773; doi: 10.1177/154193120905301202.

[7] Wanyan XR, Zhuang DM, Wei HY, Song JS. Pilot attention allocation model based on fuzzy theory. Comput Math Appl. 2011; 62(7): 2727-2735; doi: 10.1016/j.camwa.2011.06.061.

[8] Wu X, Wanyan XR, Zhuang DM. Attention allocation modeling under multi-factor condition. J Beijing Univ Aeronaut Astronaut. 2013; 39(8): 1086-1090.

[9] Ke Y, Chen L, Fu L, Jia Y, Zhao X et al. Visual attention recognition based on nonlinear dynamical parameters of EEG. Bio-Med Mater Eng. 2014; 24(1): 349-355; doi: 10.3233/BME-130817.

[10] Wilson GF, Caldwell JA, Russell CA. Performance and psychophysiological measures of fatigue effects on aviation related tasks of varying difficulty. Int J Aviat Psychol. 2007; 17(3): 219-347.

[11] Tombu M, Jolicœur P. A central capacity sharing model of dual-task performance. J Exp Psychol: Hum Percept Perform. 2003; 29(1): 3-18; doi: 10.1037/0096-1523.29.1.3.

[12] Matsui N, Bamba E. Consideration of the attention allocation problem on the basis of fuzzy entropy. Assoc Symp Meas Autom Control. 1996; 22(12): 27-32. 
[13] Wei H, Zhuang D, Wanyan X, Wang Q. An experimental analysis of situation awareness for cockpit display interface evaluation based on flight simulation. Chin J Aeronaut. 2013; 26(4): 884-889; doi: 10.1016/j.cja.2013.04.053.

[14] McKinley RA, Mclntire LK, Schmidt R, Repperger DW, Caldwell JA. Evaluation of eye metrics as a detector of fatigue. J Hum Ergon Soc. 2011; 53(4): 403-414; doi: 10.1177/0018720811411297.

[15] Langan-Fox J, Sankey MJ, Cant JM. Human factors measurement for future air traffic control systems. J Hum Ergon Soc. 2009; 51(5): 595-637; doi: 10.1177/0018720811411297.

[16] Kim HJ, Kim HS, Choi MH, Lee IH, Hong SP et al. Response time of visual matching task and heart rate in child with attention deficit hyperactivity disorder (ADHD). Bio-Med Mater Eng. 2014; 24 (1): 987-991; doi: 10.3233/BME-130895.

[17] Lavie N. Attention, distraction, and cognitive control under load. Curr Dir Psychol Sci. 2010; 19(3): 143-148; doi: 10. $1177 / 0963721410370295$.

[18] Lavie N, Hirst A, Fockert JW, Viding E. Load theory of selective attention and cognitive control. J Exp Psychol Gen. 2004; 133(3): 339-354; doi: 10.1037/0096-3445.133.3.339.

[19] Spinks JA, Zhang JX, Fox PT, Gao J, Tan LH. More workload on the central executive of working memory, less attention capture by novel visual distractors: evidence from an fMRI study. NeuroImage. 2004; 23(2): 517-524; doi: 10.1016/j.neuroimage.2004.06.025. 